\title{
Hypertension and Diabetic Kidney Disease in Children and Adolescents
}

Jane Chiang

American Diabetes Association, Alexandria, VA

Corresponding author: Jane Chiang, jchiang@diabetes.org

DOI: 10.2337/diaspect.28.3.220

(C)2015 by the American Diabetes Association. Readers may use this article as long as the work is properly cited, the use is educational and not for profit, and the work is not altered. See http:/l creativecommons.org/licenses/by-nc-nd/3.0 for details.
"I see no hope for the future of our people if they are dependent on the frivolous youth of today, for certainly all youth are reckless beyond words. When I was a boy, we were taught to be discreet and respectful of elders, but the present youth are exceedingly wise and impatient of restraint."

—Hesiod (Greek poet, 700 BC)

Y outh is a carefree time. Reckless health behavior is seldom linked to serious consequences. This is generally true for diabetes, both type 1 and type 2. For young people with diabetes, it is well known that the transition years (preteen to mid-20s) are highly problematic (1). Numerous factors compete for youths' attention, so diabetes and other health matters are low on their list of priorities. Deterioration of previously well-controlled diabetes always frustrates health care providers (HCPs) and parents, but for emerging adults trying to navigate the demands of "growing up," diabetes management naturally falls by the wayside, and rising $\mathrm{A} 1 \mathrm{C}$ levels are the proof.

Young adults tend to their diabetes management better once their lives have settled down after the tumultuous transition years. However, recent evidence is showing that, by then, complications may have developed. Although diabetes complications such as hypertension and kidney disease occur primarily in older individuals with long-standing disease, these complications are not absent in young adult and pediatric populations.

This article reviews the impact of type 1 and type 2 diabetes on long-term complications in youth, specifically focusing on hypertension and diabetic kidney disease (DKD). It concludes with considerations for future clinical research.

\section{Epidemiology: the SEARCH and TODAY Studies}

Two major studies aimed at better characterizing diabetes in youth have contributed to our understanding of the unique pathophysiology of diabetes in this population: SEARCH for Diabetes in Youth (SEARCH) and Treatment Options for Type 2 Diabetes in Adolescents and Youth (TODAY).

\section{SEARCH}

SEARCH, an ongoing, multicenter study, was a concerted effort to provide detailed epidemiological information on children and young adults, objectively characterizing the diverse populations affected by diabetes (2). SEARCH researchers identified and longitudinally tracked youths $(<20$ years of age) to assess the natural history of type 1 and type 2 diabetes, including acute and chronic complications and quality-of-life outcomes. In 2009, SEARCH estimated that 191,986 U.S. youths aged $<20$ years (up from 154,000 cases in 2001) have diabetes, including 166,984 with type 1 diabetes, 20,262 with 
type 2 diabetes, and 4,740 with other types of diabetes (2).

Between 2001 and 2009, the prevalence of type 1 diabetes in youth increased by $21.1 \%$ (95\% CI $15.6-$ 27.0), with comparable increases for both sexes and in most racial/ethnic and age-groups (3). For type 2 diabetes, the prevalence also increased significantly over the same time period by $30.5 \%$ (95\% CI 17.3-45.1), with increases observed in both boys and girls, those 10-14 and 15-19 years old, and among Hispanic, non-Hispanic white, and AfricanAmerican youths (3).

\section{TODAY}

TODAY was a multicenter, randomized clinical trial evaluating different treatment options (metformin monotherapy, metformin with a thiazolidinedione [rosiglitazone], and metformin with an intensive lifestyle intervention program) in youths 1017 years of age with type 2 diabetes.

Unlike two decades ago, type 2 diabetes is no longer uncommon in youth, and the rise of the disease tracks the growth of the obesity epidemic. According to Dabelea et al. (4), of new diagnoses of diabetes in people $<18$ years of age in the United States, one in three are type 2 diabetes, and two-thirds are type 1 diabetes. Overweight and obesity are closely linked to type 2 diabetes, with the Centers for Disease Control and Prevention reporting that $85 \%$ of adults with type 2 diabetes are also overweight or obese (5). This trend is mostly likely the same for youths.

Poor self-control is perpetuated as the root of type 2 diabetes, even by HCPs. However, the pathogenesis of type 2 diabetes is far more complex. Another widespread health assumption is that youths with poorly controlled diabetes are immune to complications, but this is also wrong. Recent evidence suggests that type 2 diabetes appears to be a more aggressive disease in youths than in adults (6-9).
Although the TODAY study was aimed at evaluating treatment options, it captured remarkable differences in the pathophysiology of diabetes between youths and adults. TODAY longitudinally tracked complications and discovered that they were more severe and developed at an accelerated rate in youths versus adults with type 2 diabetes (10). TODAY also captured the "changing faces of diabetes" (i.e., youths of various ethnic populations affected by diabetes). This study exposed the aggressive nature of diabetes in youth and showed that poor disease management during adolescence could have immediate and devastating results (6).

\section{Complications}

\section{Overall Complications}

From 1990 to 2010, strong clinical research led to steady improvements in diabetes clinical care and progressive declines in overall complication rates. The largest relative decline has been seen in acute myocardial infarctions $(-67.8 \%)$, and the smallest has been in end-stage renal disease $(-28.3 \%)(11)$. Ironically, while complication rates decreased in the adult population, youths with type 2 diabetes appeared to be experiencing higher rates and greater severity of complications with limited, if any, effective treatment options.

\section{Type 1 Diabetes}

Historically, type 1 diabetes has been more strongly associated with childhood and adolescence than has type 2 diabetes, so researchers have more aggregate data from the childhood, adolescent, and adulthood years for type 1 diabetes. This has enabled more accurate tracking of complications relative to glycemic control and disease duration in type 1 diabetes than in type 2 diabetes. Although few adolescents (aged 13-18 years) were included in the Diabetes Control and Complications Trial (DCCT) (12), both the DCCT and its 30year follow-up, the Epidemiology of Diabetes Interventions and Complications study (13), demonstrated that tight glycemic control improves long-term outcomes regardless of age. Samuelsson et al. (14) evaluated two Swedish registries ( $n=1,543$ children $)$ and validated that higher A1C levels (>8.6\% vs. $\leq 6.7 \%$ ) correlated with the presence of macroalbuminuria and retinopathy. Of note, those with higher A1C levels were also less physically active and had higher rates of smoking. Other studies have corroborated that complication risk is inversely related to improved glycemic control, which is directly linked to more sophisticated diabetes management and therapeutics $(15,16)$. In general, because of improved treatment and monitoring options, both complication rates and prognoses for youths with type 1 diabetes have improved (17).

\section{Type 2 Diabetes}

In comparison to type 1 diabetes, type 2 diabetes in youth is a relatively recent phenomenon, and the absolute numbers of young people with type 2 diabetes are modest. Therefore, longitudinal data on complications are sparse.

Type 2 diabetes is often perceived as less serious than type 1 diabetes, so initial management, treatment, and education may be less rigorous or even minimized. HCPs may recommend interminable trials of exercise and healthful eating before considering therapeutic interventions. Metformin and insulin are currently the only approved therapies for youths ( $>10$ years of age) with type 2 diabetes, so treatment options are limited, perhaps contributing to under-treatment in this population.

The comprehensive TODAY study evaluated the trajectory of hypertension (5), nephropathy (6), retinopathy (7), and cardiovascular (8) outcomes in multi-ethnic youths with type 2 diabetes and found that no therapies were particularly effective in managing disease and preventing complications. More alarming is 
that, compared to adulthood, type 2 diabetes in youth appears to be far more aggressive, is associated with more comorbidities, and has a more precipitous onset of complications, especially in those with poor glycemic control (17).

\section{Hypertension}

Type 1 Diabetes

The paradigm of arterial hypertension only plaguing adults and sparing children is no longer true. A large European study in children with type 1 diabetes $(n=2,105$, aged $5-18$ years) evaluated risk factors that led to hypertension and microalbuminuria. The authors found a clear link between the quality of metabolic control and altered blood pressure regulation and showed that age, diabetes duration, sex, BMI, A1C, and insulin dose were related to altered blood pressure profiles. They also found that youths with type 1 diabetes showed significantly increased nocturnal blood pressure (systolic blood pressure +0.51 , diastolic blood pressure +0.58 , mean arterial pressure +0.80 ), which primarily contributes to microalbuminuria (18).

\section{Type 2 Diabetes}

The U.K. Prospective Diabetes Study in adults with type 2 diabetes demonstrated that tight glycemic and blood pressure control influenced the development and progression of DKD $(19,20)$. Pinhas-Hamiel and Zeitler (21) compared rates of microalbuminuria and nephropathy in youths with type 1 versus type 2 diabetes of comparable duration and found that youths with type 2 diabetes had higher rates of microalbuminuria and worsening nephropathy. Insulin resistance likely plays a role, but this is speculative.

TODAY evaluated youths and young adults for hypertension and nephropathy and followed them for an average of 3.9 years. Subjects were enrolled within 2 years of their diagnosis (mean age 14 years) and received comprehensive diabetes care.
At baseline, $11.6 \%$ had hypertension (e.g., blood pressure $\geq 95 \%$ for age, sex, and height or blood pressure $\geq 130 / 80 \mathrm{mmHg}$ ). Initial interventions for hypertension included lifestyle modifications (dietary, exercise, and sodium education), followed by medical management (ACE inhibitor, escalated as needed). After 3.9 years of follow-up, $33.8 \%$ exhibited hypertension. The increased incidence of hypertension is most likely multifactorial in origin; inadequate adherence to lifestyle modification, behavioral influences, obesity-related factors, inadequate medical therapies, pubertal hormones, sex, and the aggressive nature of type 2 diabetes in youth all play a role. Males were at increased risk for hypertension, and hypertension risk increased by $14 \%$ per additional year of age. BMI also showed a correlation to hypertension, with a $6 \%$ increased risk for each unit increase in BMI (6).

\section{$D K D$}

DKD is defined as kidney disease attributed to diabetes (versus chronic kidney disease, which may have numerous etiologies, including diabetes). The American Diabetes Association (ADA) recommends screening for nephropathy 5 years after diagnosis for type 1 diabetes and at diagnosis for type 2 diabetes. Screening includes urine albumin excretion ( $\mathrm{mg} / \mathrm{g}$ creatinine). ADA no longer uses the terms "microalbuminuria” (30-299 mg/g creatinine) or "macroalbuminuria" $(\geq 300 \mathrm{mg} / \mathrm{g}$ creatinine), but defines albuminuria as elevated urinary albumin $(\geq 30$ $\mathrm{mg} / \mathrm{g}$ creatinine). The term "microalbuminuria" implies less serious disease, which is not true.

Glomerular filtration rate (GFR) is used to assess kidney function. Because it is difficult to measure GFR directly, the secretion of an endogenous filtration marker (i.e., serum creatinine) is used to determine the estimated GFR (eGFR). eGFR depends on age, sex, weight, and ethnicity. Younger people have higher
eGFRs because of their relatively higher muscle mass and greater average creatinine generation rate (22).

Because nephropathy is linked to hypertension, providers should closely monitor blood pressure (23). DKD may be preventable, so it is important for pediatric HCPs to understand screening procedures, risk factors, prevention measures, and treatment options.

\section{Type 1 Diabetes}

One retrospective study in pediatric type 1 diabetes found that end-stage renal disease occurred in $2.9 \%$ of its population and was significantly associated with poor glucose control (A1C $\geq 10 \%$ ), higher LDL cholesterol $(>100 \mathrm{mg} / \mathrm{dL})$, and age $>6$ years at diagnosis (24). Natural history studies in youths with type 1 diabetes suggest that structural damages to the glomeruli, interstitium, and vasculature are evident long before overt albuminuria develops, with functional changes often reflecting advanced disease (3). Although it is not entirely clear which patients are at risk for $\mathrm{DKD}$, in a large $(n=27,805)$, prospective study of youths with type 1 diabetes, Raile et al. (25) reported that $\mathrm{A} 1 \mathrm{C}$, blood pressure, dyslipidemia, diabetes duration, and male sex were correlated with development of nephropathy. The Oxford Regional Prospective Study (26) reported that microalbuminuria was associated with poor glycemic control $(30 \%$ increased risk per $1 \%$ increase in $\mathrm{A} 1 \mathrm{C}$ ) and higher GFR at 5 years $(22 \%$ increased risk per each 10 $\mathrm{mL} / \mathrm{min} / 1.73 \mathrm{~m}^{2}$ rise in GFR).

As in adulthood, albuminuria may be reversible in youth. Salardi et al. (27) retrospectively evaluated 41 youths (mean age 12.9 years) with type 1 diabetes and abnormal albumin excretion and found that $82 \%$ of untreated patients and $79 \%$ of ACE inhibitor-treated patients reverted back to normal. Thus, it appears that nephropathy may be a reversible phenomenon in youth with type 1 
diabetes, although close monitoring and treatment are still recommended.

Type 2 Diabetes

TODAY found that the prevalence of microalbuminuria tripled after $<4$ years of follow-up (6 vs. $16.6 \%$, respectively). Glycemic control contributed to the development and progression of nephropathy, with a $17 \%$ increase in albuminuria risk for each $1 \%$ increase in $\mathrm{A} 1 \mathrm{C}(6)$. Asian, African Caribbean, and American Indian (especially Pima Indian) youths are significantly affected by nephropathy. At the initial diagnosis of type 2 diabetes, $22 \%$ of Pima Indian youths had albuminuria, with an estimated prevalence of $60 \%$ by 30 years of age (28). Although type 2 diabetes is more prevalent in $\mathrm{mi}$ nority populations, race and ethnicity did not appear to have an impact on the development or progression of DKD.

\section{Treatment Options}

\section{Type 1 Diabetes}

Newer insulin analogs, continuous glucose monitoring, and continuous subcutaneous insulin infusion (insulin pump) therapy have dramatically improved care and decreased complications for youths with type 1 diabetes. Tight metabolic control, proper nutrition, exercise, tobacco-free living, and routine monitoring by HCPs remain the cornerstones of diabetes management, including minimizing complications (23). HCPs should measure A1C, blood pressure (with a correct cuff size and assessed according to age-appropriate percentiles), urine albumin-to-creatinine ratio, and serum lipids. Persistent albumin-to-creatinine ratios $>30 \mathrm{mg} / \mathrm{g}$ creatinine on three separate occasions is a useful marker of diabetic nephropathy.

An ACE inhibitor (or the angiotensin receptor II blocker (ARB) losartan for those $>6$ years of age) should be considered for treatment of hypertension or albuminuria. Postpubertal youths should be counseled about pregnancy prevention because both ACE inhibitors and ARBs may be teratogenic. There are limited longterm data on statin use in youth, but children $>10$ years of age with cardiovascular risk factors should be started on a statin. Statins should be immediately discontinued if pregnancy occurs (23).

\section{Type 2 Diabetes}

The same principles for type 1 diabetes management hold true for type 2 diabetes. Youths with a strong family history of type 2 diabetes and multiple risk factors (e.g., ethnic background, BMI, and smoking) should be provided lifestyle education (including reduced sodium intake for hypertension) and aggressively treated with medical management (including tight glycemic control and ACE inhibitors, ARBs, and statins to manage disease complications as appropriate) and tenaciously followed.

\section{Areas for Future Research}

\section{Type 1 Diabetes}

For patients with type 1 diabetes, the Adolescent Type 1 Diabetes CardioRenal Intervention Trial should shed light on the role of ACE inhibitors and statins in reducing complications, including DKD, in youth. Results are expected in December 2016.

Albuminuria is currently used to detect underlying kidney disease; however, this may be an imperfect marker. Several adult studies have shown that albuminuria may be a better marker of renal structural damage than a predictor of diabetic nephropathy risk because $50-60 \%$ of type 1 diabetes patients with albuminuria revert back to normal (29). A more accurate biomarker to predict diabetic nephropathy would be useful in this young population. It is known that hypertension and overproduction of angiotensin II exacerbate renal function. However, a better understanding of the link between hypertension and DKD would enable targeted treatment while minimizing side effects.

\section{Type 2 Diabetes}

The majority of youths with type 2 diabetes have reached puberty, with the disease rarely seen in children $<10$ years of age. The impact of pubertal hormones on the development and progression of diabetes complications remains unknown. Understanding the impact of pubertal and growth hormones on insulin resistance could facilitate the development of novel treatment options. Growth factors, pubertal hormones, inflammatory markers, and other unidentified factors may all play a role in the aggressiveness of type 2 diabetes in youth, but this remains unclear.

Treatment options for youths with type 2 diabetes are extremely limited. This is worrisome, especially because the onus of type 2 diabetes is compounded by economic hardships faced by this population. In 2014, the U.S. Food and Drug Administration approved six new therapies for adults with type 2 diabetes and none for youths (30). There are two main hurdles. First, study recruitment of this young, primarily multi-ethnic population remains extremely challenging. Finding these individuals is challenging because most youths with type 2 diabetes are from underserved, disparate communities where clinical research participation is a low priority. Regulatory authorities argue that a potential solution to the recruitment issue is a multi-therapeutic trial; sharing a single control arm (i.e., the control arm could be placebo) would have the advantage of reducing the total number of pediatric patients required (31). Second, there is a lack of consensus between industry and regulatory authorities on what constitutes a successful pathway to enable drug approval (31). The pharmaceutical industry argues that a key challenge is the regulatory requirement regarding the role of metformin and insulin in these trials, as the standard of care, which makes it virtually 
impossible to see a treatment effect from other medications (32).

Future research that would provide treatment alternatives to metformin and insulin would certainly be welcome. Although reckless behavior should never be encouraged, youths should be able to live until young adulthood to rectify their mistakes. We, as a medical community, must enable this.

\section{Duality of Interest}

No potential conflicts of interest relevant to this article were reported.

\section{Acknowledgment}

The author thanks Erika Gebel Berg, PhD, at the American Diabetes Association for superb assistance in editing the manuscript and formatting the references.

\section{References}

1. Peters A, Laffel L. Diabetes care for emerging adults: recommendations for transition from pediatric to adult diabetes care systems. Diabetes Care 2011;34:2477-2485

2. Pettitt DJ, Talton J, Dabelea D, et al., for the SEARCH for Diabetes in Youth Study Group. Prevalence of diabetes in U.S. youth in 2009: the SEARCH for Diabetes in Youth study. Diabetes Care 2014;37:402-408

3. Steinke JM, Mauer M; International Diabetic Nephropathy Study Group. Lessons learned from studies of the natural history of diabetic nephropathy in young type 1 diabetic patients. Pediatr Endocrinol Rev 2008;5(Suppl. 4):958-963

4. Dabelea D, Mayer-Davis EJ, Saydah S, et al. Prevalence of type 1 and type 2 diabetes among children and adolescents from 2001 to 2009. JAMA 2014;311:1778-1786

5. Centers for Disease Control and Prevention. Prevalence of overweight and obesity among adults with diagnosed diabetes: United States, 1988-1994 and 1999-2002. MMWR Morb Mortal Wkly Rep 2004;53:1066-1068

6. TODAY Study Group. Rapid rise in hypertension and nephropathy in youth with type 2 diabetes: the TODAY clinical trial. Diabetes Care 2013;36:1735-1741

7. TODAY Study Group. Retinopathy in youth with type 2 diabetes participating in the TODAY clinical trial. Diabetes Care 2013;36:1772-1774

8. TODAY Study Group. Lipid and inflammatory cardiovascular risk worsens over 3 years in youth with type 2 diabetes: the TODAY clinical trial. Diabetes Care 2013;36:1758-1764

9. Afkarian M, Sachs MC, Kestenbaum $\mathrm{B}$, et al. Kidney disease and increased mortality risk in type 2 diabetes. J Am Soc Nephrol 2013;24:302-308

10. TODAY Study Group; Zeitler P, Hirst K, Pyle L, et al. A clinical trial to maintain glycemic control in youth with type 2 diabetes. N Engl J Med 2012;366:2247-2256

11. Gregg EW, Li Y, Wang J, et al. Changes in diabetes-related complications in the United States, 1990-2010. N Engl J Med 2014;370:1514-1523

12. DCCT Research Group. The effect of intensive treatment of diabetes on the development and progression of long-term complications in insulin-dependent diabetes mellitus. N Engl J Med 1993;329:977-986

13. Nathan DM, Cleary PA, Backlund J-YC, et al. Intensive diabetes treatment and cardiovascular disease in patients with type 1 diabetes. N Engl J Med 2005;353:2643-2653

14. Samuelsson U, Steineck I, Gubbjornsdottir S. A high mean HbAlc value 3-15 months after diagnosis of type 1 diabetes in childhood is related to metabolic control, macroalbuminuria, and retinopathy in early adulthood: a pilot study using two nationwide population based quality registries. Pediatr Diabetes 2014;15:229-235

15. Marcovecchio ML, Dalton RN, Chiarelli F, Dunger DB. A1C variability as an independent risk factor for microalbuminuria in young people with type 1 diabetes. Diabetes Care 2011;34:1011-1013

16. Miller RG, Secrest AM, Ellis D, Becker DJ, Orchard TJ. Changing impact of modifiable risk factors on the incidence of major outcomes of type 1 diabetes: the Pittsburgh Epidemiology of Diabetes Complications Study. Diabetes Care 2013;36:3999-4006

17. Wong J, Constantino M, Yue DK. Morbidity and mortality in young-onset type 2 diabetes in comparison to type 1 diabetes: where are we now? Curr Diab Rep 2015;15:566

18. Dost A, Klinkert C, Kapellen T, et al. Arterial hypertension determined by ambulatory blood pressure profiles: contribution to microalbuminuria risk in a multicenter investigation in 2,105 children and adolescents with type 1 diabetes. Diabetes Care 2008;31:720-725

19. U.K. Prospective Diabetes Study Group. Intensive blood-glucose control with sulphonylureas or insulin compared with conventional treatment and risk of complications in patients with type 2 diabetes (UKPDS 33). Lancet 1998;352:837-853

20. U.K. Prospective Diabetes Study Group. Tight blood pressure control and risk of macrovascular and microvascular compli- cations in type 2 diabetes: UKPDS 38. BMJ 1998;317:703-713

21. Pinhas-Hamiel O, Zeitler P. Acute and chronic complications of type 2 diabetes mellitus in children and adolescents. Lancet 2007;369:1823-1831

22. National Kidney Foundation. Frequently asked questions about GFR estimates. Available from https://www. kidney.org/sites/default/files/docs/12-104004_abe_faqs_aboutgfrrevlb_singleb.pdf. Accessed 14 May 2015

23. American Diabetes Association. Microvascular complications and foot care. Sec. 9 in Standards of Medical Care in Diabetes - 2015. Diabetes Care 2015;38(Suppl. 1):S58-S66

24. Salgado PP, Silva IN, Vieira EC, Simões e Silva AC. Risk factors for early onset of diabetic nephropathy in pediatric type 1 diabetes. J Pediatr Endocrinol Metab 2010;23:1311-1320

25. Raile K, Galler A, Hofer S, et al. Diabetic nephropathy in 27,805 children, adolescents, and adults with type 1 diabetes: effect of diabetes duration, A1C, hypertension, dyslipidemia, diabetes onset, and sex. Diabetes Care 2007;30:2523-2528

26. Amin R, Turner C, van Aken S, et al. The relationship between microalbuminuria and glomerular filtration rate in young type 1 diabetic subjects: The Oxford Regional Prospective Study. Kidney Int 2005;68:1740-1749

27. Salardi S, Balsamo C, Zucchini S, et al. High rate of regression from micro-macroalbuminuria to normoalbuminuria in children and adolescents with type 1 diabetes treated or not with enalapril: the influence of HDL cholesterol. Diabetes Care 2011;34:424-429

28. Solis-Herrera C, Triplitt CL, Lynch JL. Nephropathy in youth and young adults with type 2 diabetes. Curr Diab Rep 2014:14:456

29. Perkins BA, Ficociello LH, Silva

KH, Finkelstein DM, Warram JH, Krolewski AS. Regression of microalbuminuria in type 1 diabetes. $\mathrm{N}$ Engl $\mathbf{J}$ Med 2003;348:2285-2293

30. CenterWatch. New FDA approved drugs for 2014. Available from http://www. centerwatch.com/drug-information/fdaapproved-drugs/year/2014. Accessed 20 May 2015

31. Karres J, Pratt V, Guettier J-M, et al. Joining forces: a call for greater collaboration to study new medicines in children and adolescents with type 2 diabetes. Diabetes Care 2014;37:2665-2667

32. Tamborlane WV, Klingensmith G. Crisis in care: limited treatment options for type 2 diabetes in adolescents and youth. Diabetes Care 2013;36:1777-1778 